\title{
The Laplacian Spectrum of Large Graphs Sampled from Graphons
}

\author{
Renato Vizuete, Graduate Student Member, IEEE, Federica Garin, Member, IEEE, \\ and Paolo Frasca, Senior Member, IEEE
}

\begin{abstract}
This paper studies the Laplacian spectrum and the average effective resistance of (large) graphs that are sampled from graphons. Broadly speaking, our main finding is that the Laplacian eigenvalues of a large dense graph can be effectively approximated by using the degree function of the corresponding graphon. More specifically, we show how to approximate the distribution of the Laplacian eigenvalues and the average effective resistance (Kirchhoff index) of the graph. For all cases, we provide explicit bounds on the approximation errors and derive the asymptotic rates at which the errors go to zero when the number of nodes goes to infinity. Our main results are proved under the conditions that the graphon is piecewise Lipschitz and bounded away from zero.
\end{abstract}

Index Terms-Graphons, Laplacian matrix, average effective resistance, Kirchhoff index, large networks.

\section{INTRODUCTION}

$\mathrm{T}$ HE study of large networks has been a focus of attention in recent years due to the increasing relevance of large networks in multiple fields, from computer science and engineering to biology, economics and sociology. Large networks require specific methods not only because of their size but also because their topologies are often known with large uncertainties and can dynamically evolve with time. A prominent tool to approach large networks is the concept of graphon, developed in [1], [2], [3] more than a decade ago. Graphons are infinite-dimensional representations of "families" of graphs and limit objects of convergent graph sequences. Their handy mathematical properties are allowing for a rapidly increasing number of applications in multiple fields, including game theory [4], [5], signal processing [6], |7], control theory [8], [9], and the study of diffusion processes [10] and epidemics [11], [12] on graphs. These applications are demonstrating that graphons can also be a versatile tool to study dynamics on large networks.

Since the concept of graphon is inherently related to the adjacency matrix of graphs, its applications have essentially focused on cases when the adjacency matrix is the object of study. However, this can be limiting for some applications, because many network-based dynamics are instead better described by using the Laplacian matrix of the graph [13]. Other applications of the Laplacian matrix include spectral clustering [14], combinatorial optimization [15], and signal processing [16].

The spectrum of the Laplacian matrix encodes relevant properties of the network, including its connectivity that can

R. Vizuete is with the Université Paris-Saclay, CNRS, CentraleSupelec, Laboratoire des signaux et systèmes, 91190, Gif-sur-Yvette, France, and also with the Univ. Grenoble Alpes, CNRS, Inria, Grenoble INP, GIPSA-lab, F-38000 Grenoble, France. F. Garin and P. Frasca are with Univ. Grenoble Alpes, CNRS, Inria, Grenoble INP, GIPSA-lab, F-38000 Grenoble, France. Emails: renato.vizuetedl2s.centralesupelec.fr, federica.garindinria.fr,

paolo.frascalgipsa-lab.fr.

This work has been partially supported by the French National Science Foundation through grants LabEx PERSYVAL-Lab (ANR-11-LABX-002501) and HANDY (ANR-18-CE40-0010). be measured by its spectral gap, that is, the magnitude of its smallest nonzero eigenvalue (if the spectral gap is small, the graph is poorly connected). The Laplacian spectrum also has an important role in the study of graphs by associating an electrical network to them [17]. Representing graphs as networks of resistors is a classical tool in graph theory with a large range of applications such as the analysis of random walks [18], [19], consensus algorithms [20], [21], and distributed estimation algorithms |22|. In this context, a key graph property is the average effective resistance, which can be written as a function of the eigenvalues of the Laplacian matrix. The average effective resistance, also known as Kirchhoff index, can also be used to evaluate the connectivity of a network: small values imply well-connected networks. The average effective resistance can be computed, exactly or approximately, for many specific graph topologies, including toroidal graphs [23], $d$-dimensional grids [24], and other graphs with geometric properties [20].

However, closed-form expressions for the eigenvalues are not available beyond few academic examples and therefore are of little help for graphs that represent real networks. Actually, real networks are often very large and their size can make the numerical computation of eigenvalues impractical, even accounting for the the recent developments of fast Laplacian solvers [25], [26], [27], [28]. Even worse, the topology of the network may not be fully known or be dynamically evolving, therefore preventing the direct application of numerical methods.

In this paper we demonstrate that, for large dense networks that are well described by graphons, properties of these limit objects can be used to provide useful approximations of the Laplacian properties. Work in this direction has so far been limited to spectral clustering [14], [29] and random walks on graphons [10]: we offer here a careful analysis of the approximation properties of graphons for the Laplacian eigenvalues.

In the case of large dense networks, the Laplacian matrix can be seen as a perturbation of the degree matrix of the graph [30], [31], such that the contribution of the adjacency 
matrix to the Laplacian spectrum is small. Therefore, the distribution of the eigenvalues of the Laplacian matrix is close to the distribution of the degrees. At the same time, the degree function of a graphon is closely related to the degrees of the sampled graphs [32]. Combining these two facts, it becomes natural to use the degree function of a graphon to approximate the Laplacian spectrum and, consequently, the average effective resistance of (large) graphs that are sampled from that graphon.

Motivated by this informal discussion, the objective of this paper is to use characteristics of graphons for the analysis of the spectrum of the Laplacian matrix of graphs that are sampled from graphons. More precisely, our contribution is showing that the degree function of the graphon can be used to approximate the distribution of the Laplacian eigenvalues (Theorem 1) and the average effective resistance (Theorem 2). These results will be proved under the technical assumptions of the graphon being piecewise Lipschitz and bounded away from zero.

The rest of this paper is structured as follows. Section 2 introduces the necessary preliminaries about graphons and sampled graphs. Section 3 presents our main results regarding both the Laplacian spectrum and the average effective resistance. Section 4 presents a numerical example for our results, using sequences of networks sampled from a Lipschitz continuous graphon. Finally, conclusions and future work are exposed in Section 5.

\section{GRAPHS AND GRAPHONS}

This section contains the definition of graphons and some related notions and facts that will be needed later.

\subsection{Graphons: basic notations and norms}

We begin by summarizing some definitions and results from [2], [3], [32] about kernels and graphons. The space of all bounded symmetric measurable functions $W:[0,1]^{2} \rightarrow$ $\mathbb{R}$ is denoted by $\mathcal{W}$. The elements of this space are called kernels, because of their connection with integral operators, illustrated below. The set of all kernels $W \in \mathcal{W}$ such that $0 \leq W \leq 1$ is denoted by $\mathcal{W}_{0}$ and their elements are called graphons, whose name is a contraction of graph-function. The set of all kernels $W \in \mathcal{W}$ such that $-1 \leq W \leq 1$ is denoted by $\mathcal{W}_{1}$. The degree function of a graphon is defined as:

$$
d(x):=\int_{0}^{1} W(x, y) \mathrm{d} y .
$$

We denote by $\delta_{W}$ the infimum of $d(x)$.

Every function $W \in \mathcal{W}$ defines an integral operator $T_{W}$ : $L^{2}[0,1] \rightarrow L^{2}[0,1]$ by:

$$
\left(T_{W} f\right)(x):=\int_{0}^{1} W(x, y) f(y) \mathrm{d} y .
$$

If $W$ is continuous, then $T_{W}$ is also an operator $T_{W}$ : $C[0,1] \rightarrow C[0,1]$.

For $1 \leq p<\infty$, the $L^{p}$ norm of a kernel is

$$
\|W\|_{p}:=\left(\int_{[0,1]^{2}}|W(x, y)|^{p} \mathrm{~d} x \mathrm{~d} y\right)^{1 / p}
$$

and its cut norm is

$$
\|W\|_{\square}:=\sup _{S, T \subseteq[0,1]}\left|\int_{S \times T} W(x, y) \mathrm{d} x \mathrm{~d} y\right| .
$$

For $W \in \mathcal{W}_{1}$, we have the following inequalities between $L^{p}$ norms and the cut norm [2. Equation 8.14]:

$$
\|W\|_{\square} \leq\|W\|_{1} \leq\|W\|_{2} \leq\|W\|_{1}^{1 / 2} \leq 1 .
$$

By considering the operator $T_{W}$ associated to a kernel $W \in$ $\mathcal{W}$, we can define the operator norm:

$$
\left\|T_{W}\right\|:=\sup _{\substack{f \in L^{2}[0,1] \\\|f\|_{2}=1}}\left\|T_{W} f\right\|_{2} .
$$

For the elements of $\mathcal{W}_{1}$, the cut and operator norms are related by [3, Equation 4.4 and Lemma E.6]:

$$
\|W\|_{\square} \leq\left\|T_{W}\right\| \leq \sqrt{8}\|W\|_{\square}^{1 / 2} .
$$

\subsection{Sampled Graphs}

A graphon $W$ can be used to generate a random graph with $N$ vertices, by using the following sampling method in two steps:

1. Complete Weighted Graph $\bar{G}_{N}$ : let $X=\left(X_{1}, \ldots, X_{N}\right)$ be a sequence of independent random variables uniformly distributed on the interval $[0,1]$. We generate the complete weighted graph $\bar{G}_{N}$ with $N$ vertices, whose adjacency matrix is defined as: $\bar{A}_{N}(i, j)=W\left(X_{(i)}, X_{(j)}\right)$ for all $i, j$ in $\{1, \ldots, N\}$, where $X_{(i)}$ is the $i$-th order statistic of the samples $X_{1}, \ldots, X_{N}$.

2. Simple Graph $G_{N}$ : from $\bar{G}_{N}$, we generate the simple graph $G_{N}$ with $N$ vertices by connecting each pair of distinct vertices $i \neq j$ with probability $\bar{A}_{N}(i, j)$ independently of the other edges.

The degrees of the vertices of $\bar{G}_{N}$ are denoted by $\bar{d}_{i}$ (i.e., $\bar{d}_{i}$ is the $i$ th row-sum of $\bar{A}_{N}$ ) and the normalized degrees by $\bar{\delta}_{i}=\bar{d}_{i} / N$. We introduce also the diagonal degree matrix $\bar{D}_{N}=\operatorname{diag}\left[\bar{d}_{1}, \cdots, \bar{d}_{N}\right]$ and the Laplacian matrix $\bar{L}_{N}=$ $\bar{D}_{N}-\bar{A}_{N}$. We denote the eigenvalues of $\bar{L}_{N}$ as $\bar{\lambda}_{i} \leq \cdots \leq$ $\bar{\lambda}_{N}$ and its normalized eigenvalues as $\bar{\mu}_{i}=\bar{\lambda}_{i} / N$.

Similarly, we denote the degrees of $G_{N}$ by $d_{i}$ (i.e., $d_{i}$ is the $i$ th row-sum of $A_{N}$ ) and the normalized versions by $\delta_{i}=d_{i} / N$. The degree matrix is defined as $D_{N}=\operatorname{diag}\left[d_{1}, \cdots, d_{N}\right]$ and the Laplacian matrix as $L_{N}=$ $D_{N}-A_{N}$. The eigenvalues of the Laplacian matrix are denoted by $\lambda_{1} \leq \cdots \leq \lambda_{N}$ and the normalized eigenvalues as $\mu_{i}=\lambda_{i} / N$. Notice that $\bar{A}_{N}$ is the expectation of $A_{N}$ given $X$, and hence $\bar{d}_{i}$ is the expectation of $d_{i}$ given $X$.

When needed, we will also use $\bar{d}_{(1)} \leq \cdots \leq \bar{d}_{(N)}$ to denote degrees $\bar{d}_{1}, \ldots, \bar{d}_{N}$ re-arranged in non-decreasing order, and similarly we will define $\bar{\delta}_{(i)}$ 's, $d_{(i)}$ 's and $\delta_{(i)}$ 's with a non-decreasing reordering of the corresponding (normalized) degrees.

By considering a uniform partition of $[0,1]$ into the intervals $B_{i}^{N}$, where $B_{i}^{N}=[(i-1) / N, i / N)$ for $i=1, \ldots, N-1$ and $B_{N}^{N}=[(N-1) / N, 1]$, we define the following step functions concerning degrees:

$$
d_{N}(x)=\sum_{i=1}^{N} \delta_{i} \mathbb{1}_{B_{i}^{N}}(x), \quad \tilde{d}_{N}(x)=\sum_{i=1}^{N} \delta_{(i)} \mathbb{1}_{B_{i}^{N}}(x),
$$


and the following step functions concerning Laplacian eigenvalues:

$$
\mu_{N}(x)=\sum_{i=1}^{N} \mu_{i} \mathbb{1}_{B_{i}^{N}}(x), \quad \mu_{N}^{\pi}(x)=\sum_{i=1}^{N} \mu_{\pi(i)} \mathbb{1}_{B_{i}^{N}}(x),
$$

where $\mathbb{1}_{A}(x)$ is the indicator function and $\pi \in S_{N}$, i.e., $\pi$ is a permutation of $1, \ldots, N$.

\subsection{Step Graphons Associated with Sampled Graphs}

Given a (possibly weighted) graph $G$ with $N$ vertices and with weighted adjacency matrix whose entries are $a_{i j} \in[0,1]$, the step graphon $W_{G}$ associated with $G$ is defined as

$$
W_{G}(x, y):=\sum_{i=1}^{N} \sum_{j=1}^{N} a_{i j} \mathbb{1}_{B_{i}^{N}}(x) \mathbb{1}_{B_{j}^{N}}(y)
$$

and the corresponding operator is

$$
\left(T_{W_{G}} f\right)(x):=\sum_{j=1}^{N} a_{i j} \int_{B_{j}^{N}} f(y) \mathrm{d} y \text { for any } x \in B_{i}^{N} .
$$

For a step graphon $W_{G}$ we have [2. Equation 8.15]:

$$
\left\|W_{G}\right\|_{1} \leq \sqrt{2 N}\left\|W_{G}\right\|_{\square} .
$$

To prove our main results, we will also need the following lemma, concerning the Frobenius norm $\|A\|_{F}$ of the adjacency matrix of a graph and the operator norm of the associted step graphon.

Lemma 1. Let $W$ be a step graphon associated with a graph $G$ with $N$ vertices. Then:

$$
\|A\|_{F} \leq \sqrt[4]{2 N^{5}}\left\|T_{W_{G}}\right\|^{1 / 2} .
$$

Proof. We consider the $L^{2}$ norm of the step graphon:

$$
\left\|W_{G}\right\|_{2}=\left(\int_{0}^{1} \int_{0}^{1} W_{G}^{2}(x, y) \mathrm{d} x \mathrm{~d} y\right)^{1 / 2} .
$$

We can see that $W_{G}^{2}(x, y)=W_{G}(x, y) W_{G}(x, y)$ is the product of two step functions with the same partition. Using the property $\mathbb{1}_{A \cap B}=\mathbb{1}_{A} \mathbb{1}_{B}$, we obtain:

$$
W_{G}^{2}(x, y)=\sum_{i=1}^{N} \sum_{j=1}^{N} a_{i j}^{2} \mathbb{1}_{B_{i}^{N}}(x) \mathbb{1}_{B_{j}^{N}}(y),
$$

and hence:

$$
\begin{aligned}
& \left\|W_{G}\right\|_{2}^{2}=\int_{0}^{1} \int_{0}^{1} \sum_{i=1}^{N} \sum_{j=1}^{N} a_{i j}^{2} \mathbb{1}_{B_{i}^{N}}(x) \mathbb{1}_{B_{j}^{N}}(y) \mathrm{d} x \mathrm{~d} y \\
= & \sum_{i=1}^{N} \sum_{j=1}^{N} \int_{\frac{i-1}{N}}^{\frac{i}{N}} \int_{\frac{j-1}{N}}^{\frac{j}{N}} a_{i j}^{2} \mathrm{~d} x \mathrm{~d} y=\frac{1}{N^{2}} \sum_{i=1}^{N} \sum_{j=1}^{N} a_{i j}^{2}=\frac{\|A\|_{F}^{2}}{N^{2}} .
\end{aligned}
$$

This gives $\|A\|_{F}=N\left\|W_{G}\right\|_{2}$. Finally, using (1), (2), (3) implies $\|A\|_{F} \leq N\left\|W_{G}\right\|_{1}^{1 / 2} \leq \sqrt[4]{2 N^{5}}\left\|W_{G}\right\|_{\square}^{1 / 2} \leq$ $\sqrt[4]{2 N^{5}}\left\|T_{W_{G}}\right\|^{1 / 2}$.

For the graphs $\bar{G}_{N}$ and $G_{N}$ sampled from a graphon $W$ as described in Section 2.2, we will denote the corresponding step graphons with the short-hand notations $\bar{W}_{N}:=W_{\bar{G}_{N}}$ and $W_{N}:=W_{G_{N}}$.

\subsection{Graphs Sampled from Piecewise Lipschitz Graphons}

We shall restrict our analysis to a class of graphons that is wide enough to be relevant for the applications, but leads to a tractable analysis. We therefore consider the class of piecewise Lipschitz graphons, some properties of which we recall from [32].

Definition 2.1 (Piecewise Lipschitz graphon). Graphon $W$ is said to be piecewise Lipschitz if there exists a constant $L$ and a sequence of non-overlapping intervals $I_{k}=\left[\alpha_{k-1}, \alpha_{k}\right)$ defined by $0=\alpha_{0}<\cdots<\alpha_{K+1}=1$, for a finite nonnegative integer $K$ such that for any $k, \ell$, any set $I_{k \ell}=$ $I_{k} \times I_{\ell}$ and pairs $\left(x_{1}, y_{1}\right)$ and $\left(x_{2}, y_{2}\right) \in I_{k \ell}$ we have that:

$$
\left|W\left(x_{1}, y_{1}\right)-W\left(x_{2}, y_{2}\right)\right| \leq L\left(\left|x_{1}-x_{2}\right|+\left|y_{1}-y_{2}\right|\right) .
$$

If $K=0$, then the graphon is said to be Lipschitz.

Notice that when $W$ is a piecewise Lipschitz graphon, the degree function $d(x)$ is piecewise continuous, and hence $\delta_{W}$ is its minimum, and not just its infimum.

Definition 2.2 (Large enough $N$ ). Given a piecewise Lipschitz graphon $W$ and $\nu<e^{-1}, N$ is large enough if $N$ satisfies the following conditions:

$$
\begin{gathered}
\frac{2}{N}<\min _{k \in\{1, \ldots, K+1\}}\left(\alpha_{k}-\alpha_{k-1}\right), \\
\frac{1}{N} \log \left(\frac{2 N}{\nu}\right)+\frac{1}{N}(2 K+3 L)<\max _{x} d_{W}(x), \\
N e^{-N / 5}<\nu .
\end{gathered}
$$

The following result is given in [32] as Theorems 1 and 2.

Lemma 2. For a piecewise Lipschitz graphon $W$ and $N$ large enough, with probability at least $1-\nu$ :

$$
\begin{gathered}
\left\|T_{\bar{W}_{N}}-T_{W}\right\| \mid \leq 2 \sqrt{\left(L^{2}-K^{2}\right) b_{N}^{2}+K b_{N}}=: \vartheta(N), \\
\left\|\bar{d}_{N}(x)-d(x)\right\|_{2} \leq \vartheta(N),
\end{gathered}
$$

and with probability at least $1-2 \nu$ :

$$
\begin{gathered}
\left\|T_{W_{N}}-T_{W}\right\| \leq \sqrt{\frac{4 \log (2 N / \nu)}{N}}+\vartheta(N)=: \phi(N), \\
\left\|d_{N}(x)-d(x)\right\|_{2} \leq \phi(N),
\end{gathered}
$$

where $b_{N}:=\frac{1}{N}+\sqrt{\frac{8 \log (N / \nu)}{N+1}}$.

From Lemma 2 we can see that the constant $\nu$ determines the probability with which the results hold, such that if we want a higher probability, the value of $N$ will increase to satisfy the large enough condition. The constant $\nu$ will appear in most of the results of the paper.

To obtain the main results of our paper (see Section 3 we will consider graphons which are piecewise Lipschitz. Moreover, when needed, we will consider graphons which are bounded away from zero, i.e., whose infimum (denoted by $\eta_{W}$, and which is actually a minimum under the piecewise Lipschitz assumption) is strictly positive. Graphons which are bounded away from zero are also known as graphons having 'minimal degree' [32], since the assumption $W(x, y) \geq \eta_{W}>0$ for all $x, y$ has the following 
implications about the degrees, both of the graphon itself and of the graphs sampled from the graphon: $\delta_{W} \geq \eta_{W}>0$ and $\bar{\delta}_{i} \geq \eta_{W}$ for all $i=1, \ldots, N$.

\subsection{Laplacian Operator of a Graphon}

The Laplacian matrix of a graph is defined as the difference between the degree matrix and the adjacency matrix. In analogy with this definition, we can define a Laplacian operator for graphons $\mathcal{L}_{W}: L^{2}[0,1] \rightarrow L^{2}[0,1]$ as:

$$
\left(\mathcal{L}_{W} f\right)(x):=d(x) f(x)-\left(T_{W} f\right)(x) .
$$

If the graphon is continuous, $\mathcal{L}_{W}$ is also an operator in the space of continuous functions $\mathcal{L}_{W}: C[0,1] \rightarrow C[0,1]$, see [14|. The spectrum of this operator is composed by an essential spectrum located in the range of the degree function $d(x)$ and a finite number of isolated eigenvalues $\kappa_{i}$, which can only have accumulation points in the boundaries of the essential spectrum. The isolated eigenvalues are contained in the interval $[0,1]$ and $\kappa_{1}=0$ is always an eigenvalue with a constant eigenfunction associated $\psi_{1}(x)=k$.

\section{Main RESUltS ON LAPLACIAN SPECTRUM}

This section contains our main results about the Laplacian spectrum, which regard the whole distribution of the eigenvalues (Section 3.1), the spectral gap (Section 3.2) and the average effective resistance (Section 3.3). We conclude the section with some remarks about an easy extension of our results to deterministically sampled graphs (Section 3.4 .

\subsection{Distribution of eigenvalues}

For a large dense network, the distribution of the eigenvalues of the Laplacian matrix is close to the distribution of the degrees of the vertices [31]. Using results of perturbation theory, [30| derived a bound for the relative error in the estimation of the eigenvalues of the Laplacian matrix using the degrees of the network for simple graphs:

$$
\frac{\left\|\lambda_{G}-\tilde{d}_{G}\right\|_{2}}{\left\|\tilde{d}_{G}\right\|_{2}} \leq \sqrt{\frac{N}{\left\|\tilde{d}_{G}\right\|_{1}}}
$$

where $\lambda_{G}$ is a vector with the Laplacian eigenvalues arranged in non-decreasing order and $\tilde{d}_{G}$ is a vector with the degrees of the network arranged in non-decreasing order. In particular, for a sequence of graphs where at least a constant fraction of vertices have a degree growing linearly with $N$, the right-hand side of (10) decays to zero as $O(1 / \sqrt{N})$.

Graphs $G_{N}$ sampled from a graphon as described in Section 2.2 are dense graphs, and it is natural to look for an analogous of (10), so as to show that the eigenvalues of the Laplacian of $G_{N}$ are mostly determined by the reordered degree function of the same graph, with an error bounded by a quantity only depending on $N$ and on the graphon (see Proposition 1.

Proposition 1. For a piecewise Lipschitz graphon $W$ and $N$ large enough, with probability at least $1-2 \nu$ :

$$
\left\|\mu_{N}(x)-\tilde{d}_{N}(x)\right\|_{2} \leq \sqrt[4]{\frac{2}{N}} \sqrt{\left\|T_{W}\right\|+\phi(N)},
$$

with $\phi(N)$ as in Lemma 2
Proof. By definition:

$$
\begin{aligned}
& \left\|\mu_{N}(x)-\tilde{d}_{N}(x)\right\|_{2}^{2}=\int_{0}^{1} \sum_{i=1}^{N}\left|\mu_{i}-\delta_{(i)}\right|^{2} \mathbb{1}_{B_{i}^{N}}(x) \mathrm{d} x \\
& =\frac{1}{N} \sum_{i=1}^{N}\left|\mu_{i}-\delta_{(i)}\right|^{2}=\frac{1}{N^{3}} \sum_{i=1}^{N}\left|\lambda_{i}-d_{(i)}\right|^{2} .
\end{aligned}
$$

We can use the Wielandt-Hoffman Theorem [33], obtaining:

$$
\sum_{i=1}^{N}\left|\lambda_{i}-d_{(i)}\right|^{2} \leq\left\|A_{N}\right\|_{F}^{2}
$$

and hence

$$
\left\|\mu_{N}(x)-\tilde{d}_{N}(x)\right\|_{2} \leq \frac{1}{N^{3 / 2}}\left\|A_{N}\right\|_{F} .
$$

By using Lemma 1 we get:

$$
\left\|\mu_{N}(x)-\tilde{d}_{N}(x)\right\|_{2} \leq \frac{\sqrt[4]{2 N^{5}}\left\|T_{W_{N}}\right\|^{1 / 2}}{N^{3 / 2}} .
$$

Finally we notice that $\left\|T_{W_{N}}\right\| \leq\left|\left\|T_{W_{N}}-T_{W}\right\|\right|+\left\|T_{W}\right\| \mid$ and we use (7) from Lemma 2 to obtain the desired result.

Furthermore, we can approximate the distribution of the normalized Laplacian eigenvalues by using the degree function of the graphon, as follows.

Proposition 2. For a piecewise Lipschitz graphon $W$ and $N$ large enough, with probability at least $1-2 \nu$ :

$$
\min _{\pi \in S_{N}}\left\|\mu_{N}^{\pi}(x)-d(x)\right\|_{2} \leq \sqrt[4]{\frac{2}{N}} \sqrt{\left\|T_{W}\right\|+\phi(N)}+\phi(N),
$$

with $\phi(N)$ as in Lemma 2

Proof. In addition to the step functions defined in Section 2.2 in this proof we will also use:

$$
\bar{\mu}_{N}^{\pi}(x)=\sum_{i=1}^{N} \bar{\mu}_{\pi(i)} \mathbb{1}_{B_{i}^{N}}(x) .
$$

The goal of this proof is to show that, with probability at least $1-2 \nu$, there exists a permutation $\sigma$ such that:

$$
\left\|\mu_{N}^{\sigma}(x)-d(x)\right\|_{2} \leq \sqrt[4]{\frac{2}{N}} \sqrt{\left\|T_{W}\right\|+\phi(N)}+\phi(N) .
$$

Notice that a different $\sigma$ might be used for different realizations of the random graph $G_{N}$.

By applying the triangle inequality in $\left\|\mu_{N}^{\sigma}(x)-d(x)\right\|_{2}$, we get that, for any $\sigma$ :

$$
\left\|\mu_{N}^{\sigma}(x)-d(x)\right\|_{2} \leq\left\|\mu_{N}^{\sigma}(x)-d_{N}(x)\right\|_{2}+\left\|d_{N}(x)-d(x)\right\|_{2} .
$$

For the first term, we have:

$$
\left\|\mu_{N}^{\sigma}(x)-d_{N}(x)\right\|_{2}=\frac{1}{N^{3 / 2}}\left(\sum_{i=1}^{N}\left|\lambda_{\sigma(i)}-d_{i}\right|^{2}\right)^{1 / 2} .
$$

Then, we apply Wielandt-Hoffman theorem to $A_{N}=D_{N}-$ $L_{N}$, which gives:

$$
\min _{\pi \in S_{N}} \sum_{i=1}^{N}\left|\lambda_{\pi(i)}-d_{i}\right|^{2} \leq\left\|A_{N}\right\|_{F}^{2} .
$$


We choose $\sigma$ to be the permutation that achieves the above minimum, so that we get:

$$
\left\|\mu_{N}^{\sigma}(x)-d_{N}(x)\right\|_{2} \leq \frac{\left\|A_{N}\right\|_{F}}{N^{3 / 2}} .
$$

For an upper bound on $\left\|A_{N}\right\|_{F}$, we apply Lemma 1, and then we apply the inequality $\left\|T_{W_{N}}\right\| \leq\|\| T_{W_{N}}-T_{W} \|+$ $\left\|T_{W}\right\|$ and the bound (7) from Lemma 2. We obtain that with probability at least $1-\nu$ there exists $\sigma$ such that:

$$
\left\|\mu_{N}^{\sigma}(x)-d_{N}(x)\right\|_{2} \leq \sqrt[4]{\frac{2}{N}} \sqrt{\left\|T_{W}\right\|+\phi(N)} .
$$

For the second term in the right-hand side of (11), we use (8) from Lemma 2. Notice that Lemma 2 ensures that with probability at least $1-\nu$ both bounds (7) and (8) hold true, together; this ensures that with the same probability both bounds (12) and (8) hold true, together.

Proposition 2 is key in our analysis because it makes the connection between graphs and graphons: the result ensures that the degree function of the graphon provides a good approximation of the eigenvalues of the graph.

The statement of Proposition 2 involves finding the best re-ordering $\pi$ of the Laplacian eigenvalues, so as to minimize $\left\|\mu_{N}^{\pi}(x)-d(x)\right\|_{2}$ : if we want to know the function $\mu_{N}^{\pi}(x)$ that satisfies the corresponding upper bounds, it is necessary to evaluate $N$ ! possible permutations. A simpler statement can be obtained by adding a suitable monotonicity assumption to a graphon bounded away from zero. In the theorem below we will consider a graphon $W$ that is bounded away from zero and is non-decreasing, i.e., such that $W\left(x_{1}, y\right) \leq W\left(x_{2}, y\right)$ when $x_{1} \leq x_{2}$. A graphon being non-decreasing implies that its degree function is also nondecreasing, even though the converse is not true.

Theorem 1. For a piecewise Lipschitz, non-decreasing graphon with minimum $\eta_{W}>0$ and for $N$ large enough, with probability at least $1-3 \nu$ :

$\left\|\mu_{N}(x)-d(x)\right\|_{2} \leq \varphi(N)+\sqrt[4]{\frac{2}{N}} \sqrt{\left\|T_{W}\right\|+\vartheta(N)}+\vartheta(N)$, with $\vartheta(N)$ as in Lemma 2 and $\varphi(N)$ as in Lemma 3 below.

To prove Theorem 1, we first need the following concentration results for the normalized degrees of $G_{N}$ and $\bar{G}_{N}$ and for the normalized eigenvalues of the corresponding Laplacian matrices.

Lemma 3. Given a graphon $W$ with infimum $\eta_{W}>0$, if $N$ is large enough, with probability at least $1-\nu$ the normalized degrees of the graphs $G_{N}$ and $\bar{G}_{N}$ sampled from $W$ satisfy:

$$
\max _{i=1, \ldots, N}\left|\delta_{(i)}-\bar{\delta}_{(i)}\right| \leq \sqrt{\frac{\log (2 N / \nu)}{N \eta_{W}}}:=\gamma(N),
$$

and with probability at least $1-2 \nu$ the normalized eigenvalues of their Laplacian matrices $L_{N}$ and $\bar{L}_{N}$ satisfy:

$$
\max _{i=1, \ldots, N}\left|\mu_{i}-\bar{\mu}_{i}\right| \leq\left(\frac{1}{\sqrt{\eta_{W}}}+2\right) \sqrt{\frac{\log (2 N / \nu)}{N}}:=\varphi(N) .
$$

Proof. For the first part of the proof, we use Chernoff bound, as in [34. Proof of Theorem 2], thanks to the remark that $\bar{d}_{i}$ is the expectation of $d_{i}$, conditioned on $X$, and that $d_{i}$ is the $i$ th row-sum of $A_{N}$. By the Chernoff bound, for any given $i$ :

$$
\operatorname{Pr}\left[\left|d_{i}-\bar{d}_{i}\right|>b \bar{d}_{i}\right] \leq \frac{\nu}{N} \quad \text { if } \quad b \geq \sqrt{\frac{\log (2 N / \nu)}{\bar{d}_{i}}}
$$

Since $\bar{d}_{i} \geq N \eta_{W}$, by considering $b=\sqrt{\frac{\log (2 N / \nu)}{N \eta_{W}}}$, for any given $i$, we have with probability at least $1-\nu / N$ :

$$
\left|d_{i}-\bar{d}_{i}\right| \leq \sqrt{\frac{\log (2 N / \nu)}{N \eta_{W}}} \bar{d}_{i} \leq \sqrt{\frac{\log (2 N / \nu)}{N \eta_{W}}} \bar{d}_{(N)}
$$

Hence, with probability at least $1-\nu$ this bound is true for all $i=1, \ldots, N$. Since $D_{N}-\bar{D}_{N}$ is diagonal,

$$
\left\|D_{N}-\bar{D}_{N}\right\|_{2}=\max _{i=1, \ldots, N}\left|d_{i}-\bar{d}_{i}\right| \leq \sqrt{\frac{\log (2 N / \nu)}{N \eta_{W}}} \bar{d}_{(N)}
$$

with probability at least $1-\nu$.

From Weyl's Theorem, $\max _{i}\left|d_{(i)}-\bar{d}_{(i)}\right| \leq\left\|D_{N}-\bar{D}_{N}\right\|_{2}$, which ends the proof of (13), recalling that $\bar{d}_{(N)} \leq N, d_{(i)}=$ $N \delta_{(i)}$ and $\bar{d}_{(i)}=N \bar{\delta}_{(i)}$.

For the second part of the lemma, we have:

$$
\begin{aligned}
\left\|L_{N}-\bar{L}_{N}\right\|_{2} & =\left\|D_{N}-A_{N}-\bar{D}_{N}+\bar{A}_{N}\right\|_{2} \\
& \leq\left\|D_{N}-\bar{D}_{N}\right\|_{2}+\left\|\bar{A}_{N}-A_{N}\right\|_{2} .
\end{aligned}
$$

From [34, Theorem 1], we have that with probability at least $1-\nu$ :

$$
\left\|A_{N}-\bar{A}_{N}\right\|_{2} \leq \sqrt{4 \bar{d}_{(N)} \log (2 N / \nu)} .
$$

By combining (15) and (16) we have with probability at least $1-2 \nu$ :

$$
\left\|L_{N}-\bar{L}_{N}\right\|_{2} \leq \sqrt{\frac{\bar{d}_{(N)}^{2} \log (2 N / \nu)}{N \eta_{W}}}+\sqrt{4 \bar{d}_{(N)} \log (2 N / \nu)} .
$$

By using Weyl's Theorem and considering the normalized eigenvalues we get:

$$
\max _{i}\left|\mu_{i}-\bar{\mu}_{i}\right| \leq \sqrt{\frac{\bar{d}_{(N)}^{2} \log (2 N / \nu)}{N^{3} \eta_{W}}}+\sqrt{\frac{4 \bar{d}_{(N)} \log (2 N / \nu)}{N^{2}}} .
$$

Finally, since $\bar{d}_{(N)} \leq N$, we get the desired result.

The proof of Theorem 1 and the proof of Theorem 2 in the next section heavily rely both on Lemma 2 and on Lemma 3 More precisely, each proof will require the simultaneous use of various bounds from such lemmas; since each bound holds with some probability, the following lemma is about the joint probability of the bounds of interest.

Lemma 4. Given a piecewise Lipschitz graphon $W$ with infimum $\eta_{W}>0$, if $N$ is large enough, then:

- with probability $1-3 \nu$, the three bounds (5), (6) and (14) hold true;

- with probability $1-3 \nu$, the four bounds (7), 8, , 13) and (14) hold true.

Proof. The first statement is immediately obtained: Lemma 2 ensures that with probability at least $1-\nu$ both (5) and (6) hold true, while Lemma 3 ensures that (14) holds true with probability at least $1-2 \nu$. Hence, the probability that all three bounds hold true is at least $1-\nu-2 \nu=1-3 \nu$. 
For the second statement, we need a closer look at the proofs of Lemmas 2 and 3 . The same event (15), which has probability at least $1-\nu$, is used in the proof of both statements (13) and (14) of Lemma 3 Hence, the probability that both bounds hold together is at least $1-2 \nu$. Moreover, the same event [16, which has probability at least $1-\nu$ thanks to [34. Theorem 1], is used both in the above proof of Lemma 3 and in the proof of the two statements (7) and (8) in Lemma 2 (see [32. Proof of Theorem 1] for the latter proof). Hence, the probability that all the bounds [13), (14), (7), and (8) hold true together is at least $1-3 \nu$.

Now we have all the tools needed to prove Theorem 1 .

Proof of Theorem 11 In this proof, we will make use of the bounds (5), 6) and (14) from Lemmas 2 and 3 ; by Lemma 4 the event that all three bounds hold true has probability at least $1-3 \nu$.

We start from the left-hand side of the claimed inequality, and we use the triangle inequality to obtain that:

$$
\begin{aligned}
\| \mu_{N}(x)- & d(x)\left\|_{2} \leq\right\| \mu_{N}(x)-\bar{\mu}_{N}(x) \|_{2} \\
& +\left\|\bar{\mu}_{N}(x)-\bar{d}_{N}(x)\right\|_{2}+\left\|\bar{d}_{N}(x)-d(x)\right\|_{2} .
\end{aligned}
$$

The first term can be rewritten and bounded by using (14):

$$
\left\|\mu_{N}(x)-\bar{\mu}_{N}(x)\right\|_{2}=\left(\frac{1}{N} \sum_{i=1}^{N}\left|\mu_{i}-\bar{\mu}_{i}\right|^{2}\right)^{1 / 2} \leq \varphi(N) .
$$

For the second term, we have:

$$
\left\|\bar{\mu}_{N}(x)-\bar{d}_{N}(x)\right\|_{2}=\frac{1}{N^{3 / 2}}\left(\sum_{i=1}^{N}\left|\bar{\lambda}_{i}-\bar{d}_{i}\right|^{2}\right)^{1 / 2} .
$$

By applying Wielandt-Hoffman theorem to $\bar{A}_{N}=\bar{D}_{N}-\bar{L}_{N}$, we obtain:

$$
\min _{\pi \in S_{N}} \sum_{i=1}^{N}\left|\bar{\lambda}_{\pi(i)}-\bar{d}_{i}\right|^{2} \leq\left\|\bar{A}_{N}\right\|_{F}^{2} .
$$

The assumption that $W$ is non-decreasing implies that also $\bar{d}_{N}(x)$ is non-decreasing, i.e., $\bar{d}_{1} \leq \cdots \leq \bar{d}_{N}$. Indeed, since $\lambda_{1} \leq \cdots \leq \lambda_{N}$ by definition and $\bar{d}_{1} \leq \cdots \leq \bar{d}_{N}$ thanks to the monotonicity assumption, the minimum in 19] (Wielandt-Hoffman theorem) is achieved for the identity permutation that leaves all positions unchanged. Then we obtain:

$$
\left\|\bar{\mu}_{N}(x)-\bar{d}_{N}(x)\right\|_{2}=\frac{1}{N^{3 / 2}}\left(\sum_{i=1}^{N}\left|\bar{\lambda}_{i}-\bar{d}_{i}\right|^{2}\right)^{1 / 2} \leq \frac{\left\|\bar{A}_{N}\right\|_{F}}{N^{3 / 2}} .
$$

Then, we can use Lemma 1, and the inequality $\left\|T_{\bar{W}_{N}}\right\| \leq \leq$ $\left\|T_{\bar{W}_{N}}-T_{W}\right\|\|+\| T_{W} \|$ with the bound (5) to obtain that

$$
\left\|\bar{\mu}_{N}(x)-\bar{d}_{N}(x)\right\|_{2} \leq \sqrt[4]{\frac{2}{N}} \sqrt{\left\|T_{W}\right\|+\vartheta(N)} .
$$

For the third term in the right-hand side of (17), we use (6).

Finally, the desired result is obtained from (17) by combining the three bounds (18), 20, and (6).

Theorem 1 has implications on the asymptotic behavior of $\left\|\mu_{N}(x)-d(x)\right\|_{2}$, which are discussed in the next remark.

Remark 1. The upper bound on $\left\|\mu_{N}(x)-d(x)\right\|_{2}$ in Theorem 1 holds true with probability at least $1-3 \nu$, and has an expression which depends both on $\nu$ and on $N$. We are interested in its asymptotic behaviour for $N \rightarrow \infty$. When we consider a constant $\nu$, we can easily see that this upper bound goes to zero as $O\left((\log (N) / N)^{1 / 4}\right)$, since $\varphi(N)=O\left((\log (N) / N)^{1 / 2}\right)$ and $\vartheta(N)=O\left((\log (N) / N)^{1 / 4}\right)$; moreover, if the graphon is Lipschitz $(K=0)$, then the upper bound goes to zero as $O\left((1 / N)^{1 / 4}\right)$, since in this case $\vartheta(N)=O\left((\log (N) / N)^{1 / 2}\right)$. It is interesting to notice that all these asymptotic behaviours remain the same also when we consider $\nu=1 / N^{\alpha}$ for any positive constant $\alpha$, since this only affects constant factors. By choosing $\alpha>1$, we can then apply Borel-Cantelli Lemma and obtain that, under the assumptions of Thm. 1. almost surely $\left\|\mu_{N}(x)-d(x)\right\|_{2}$ decays to zero as $O\left((\log (N) / N)^{1 / 4}\right)$, and under the further assumption that the graphon is Lipschitz then almost surely $\left\|\mu_{N}(x)-d(x)\right\|_{2}$ goes to zero as $O\left((1 / N)^{1 / 4}\right)$.

Similarly, from Prop. 2 we obtain that for any piecewise Lipschitz graphon almost surely $\min _{\pi \in S_{N}}\left\|\mu_{N}^{\pi}(x)-d(x)\right\|_{2}$ decays to zero as $O\left((\log (N) / N)^{1 / 4}\right)$, and for any Lipschitz graphon almost surely $\min _{\pi \in S_{N}}\left\|\mu_{N}^{\pi}(x)-d(x)\right\|_{2}$ decays to zero as $O\left((1 / N)^{1 / 4}\right)$.

\subsection{Spectral gap}

The results in Section 3.1 concern the distribution of eigenvalues of the Laplacian matrix. However, it is often useful to obtain more detailed information on small eigenvalues, and in particular on the second largest, to see its distance from zero. This distance, also known as the spectral gap, is a measure of how well connected is the graph and plays a crucial role in shaping the properties of graph-based dynamics such as random walks on graphs and consensusseeking systems [35], [36], [37].

In this section we give some results on $\bar{\mu}_{2}$, the spectral gap of $\bar{G}_{N}$, and on $\mu_{2}$, the spectral gap of $G_{N}$ : the results about $\bar{\mu}_{2}$ will become useful in the next section. The two spectral gaps are closely related, due to Lemma 3.

Remark 2. Given a graphon $W$ with infimum $\eta_{W}>0$, by Lemma 3 we have $\left|\mu_{2}-\bar{\mu}_{2}\right| \leq \varphi(N)$ with probability at least $1-2 \nu$. By taking $\nu=1 / N^{\alpha}$ for some $\alpha>1$, we obtain that $\left|\mu_{2}-\bar{\mu}_{2}\right|=O\left((\log (N) / N)^{1 / 2}\right)$ with probability at least $1-O\left(1 / N^{\alpha}\right)$, and hence we can apply Borel-Cantelli Lemma to conclude that almost surely $\left|\mu_{2}-\bar{\mu}_{2}\right|$ decays to zero as $O\left((\log (N) / N)^{1 / 2}\right)$.

Lemma 5. For a complete weighted graph sampled from a graphon with infimum $\eta_{W}$ :

$$
\bar{\mu}_{2} \geq \eta_{W}
$$

Proof. We use the variational characterization of eigenvalues (Courant-Fischer theorem). Since $\bar{\lambda}_{1}=0$ with eigenvector $1_{N}$ (the all-ones vector of size $N$ ),

$$
\bar{\mu}_{2}=\frac{1}{N} \bar{\lambda}_{2}=\frac{1}{N} \min _{\substack{x: x^{T} x=1 \\ x^{T} 1_{N}=0}} x^{T} \bar{L}_{N} x .
$$

Since $\bar{L}_{N}$ is a symmetric Laplacian matrix,

$x^{T} \bar{L}_{N} x=\frac{1}{2} \sum_{i} \sum_{j} \bar{A}_{N}(i, j)\left(x_{i}-x_{j}\right)^{2} \geq \frac{\eta_{W}}{2} \sum_{i} \sum_{j}\left(x_{i}-x_{j}\right)^{2}$.

Then notice that $\sum_{i} \sum_{j}\left(x_{i}-x_{j}\right)^{2}=2 N\left[\left(\sum_{i} x_{i}^{2}\right)-\left(\sum_{i} x_{i}\right)^{2}\right]$ and hence for all $x$ such that $x^{T} x=1$ and $x^{T} 1_{N}=0$ we 
have $\sum_{i} \sum_{j}\left(x_{i}-x_{j}\right)^{2}=2 N$, so that $x^{T} \bar{L}_{N} x \geq N \eta_{W}$. With this, together with (21), we can conclude that $\bar{\mu}_{2} \geq \eta_{W}$.

By combining (14) and Lemma 5 we obtain the following lower bound for $\mu_{2}$.

Proposition 3. For a simple graph sampled from a graphon with infimum $\eta_{W}$ and for $N$ large enough, with probability at least $1-2 \nu$ :

$$
\mu_{2} \geq \eta_{W}-\varphi(N), \text { with } \varphi(N) \text { as in (14). }
$$

From Proposition 3 we can see that a sufficient condition to guarantee that the spectral gap $\mu_{2}$ remains bounded away from zero a.s. is having a graphon with $\eta_{W}>0$. Another case in which $\mu_{2}$ is guaranteed to remain bounded away from zero a.s. is given by Proposition 4 below (based on the results in |14|): when the graphon $W$ is continuous, has $\delta_{W}>0$ and its zero eigenvalue $\kappa_{1}=0$ has multiplicity one.

Proposition 4. Let $W$ be a continuous graphon. Let $M$ be the number of isolated eigenvalues of $W$ in the interval $\left[0, \delta_{W}\right]$, counted with their multiplicities, and let $0=\kappa_{1} \leq \kappa_{2} \leq \cdots \leq$ $\kappa_{M}$ be such eigenvalues. Define $\rho=\kappa_{2}$ if $M \geq 2$, and $\rho=\delta_{W}$ if $M=1$. Then,

$$
\lim _{N \rightarrow \infty} \mu_{2}=\rho, \quad \text { a.s. }
$$

Proof. The results in [14] imply that $\lim _{N \rightarrow \infty} \bar{\mu}_{2}=\rho$ a.s., as we show below. Consider the operator $\mathcal{L}_{P_{N}}: C[0,1] \rightarrow$ $C[0,1]$ :

$$
\left(\mathcal{L}_{P_{N}} f\right)(x):=d_{P_{N}}(x) f(x)-\int_{0}^{1} W(x, y) f(y) \mathrm{d} P_{N}(y),
$$

where $d_{P_{N}}(x):=\int_{0}^{1} W(x, y) \mathrm{d} P_{N}(y), P_{N}:=1 / N \sum_{i=1}^{N} \delta_{X_{i}}$ is the empirical distribution and $\delta_{X_{i}}$ is the Dirac measure. Isolated eigenvalues of $\mathcal{L}_{P_{N}}$ are also eigenvalues of $\bar{L}_{N}$ Proposition 22]. According to [14. Proposition 23], $\mathcal{L}_{P_{N}}$ converges compactly to $\mathcal{L}_{W}$ a.s., which implies the convergence of isolated parts of the spectrum and due to the uppersemicontinuity, the limits of convergent sequences are the isolated eigenvalues of $\mathcal{L}_{W}$. Additionally, [14. Proposition 6] implies that for an isolated eigenvalue $\kappa$ with multiplicity $m$, there are $m$ sequences of eigenvalues that converge to $\kappa$. If $\kappa_{1}=0$ has multiplicity 1 and there are no isolated eigenvalues in the interval $\left(0, \delta_{W}\right)$, then the second eigenvalue $\bar{\mu}_{2}$ will converge to $\delta_{W}$ according to [14, Proposition 24].

Finally, by Remark 2 we have that almost surely $\lim _{N \rightarrow \infty} \mu_{2}=\lim _{N \rightarrow \infty} \bar{\mu}_{2}$, which completes the proof.

Notice that Proposition 4 requires the additional assumption that the graphon is continuous, which was not required in Proposition 3 , but on the other hand it does not require the graphon to be bounded away from zero and moreover it gives a much richer result, since it characterizes the almost sure limit of $\mu_{2}$ for $N \rightarrow \infty$.

\subsection{Average effective resistance}

We consider the simple graph $G_{N}$ as an electrical network where all the edges have resistance equal to 1 . Between two vertices $i$ and $j$, we denote the effective resistance $R_{\text {eff }}(i, j)$ as the electrical potential difference induced between $i$ and $j$ by a unit current injected in $i$ and extracted from $j$. The average effective resistance of $G_{N}$ is defined as:

$$
R_{N}^{\mathrm{ave}}:=\frac{1}{2 N^{2}} \sum_{i=1}^{N} \sum_{j=1}^{N} R_{\mathrm{eff}}(i, j)
$$

This quantity is also related to the spectrum of the Laplacian matrix of the graph [37]:

$$
R_{N}^{\text {ave }}=\frac{1}{N} \sum_{i=2}^{N} \frac{1}{\lambda_{i}} .
$$

This handy characterization immediately leads to draw some conclusions about its asymptotic behavior.

Remark 3. To find the asymptotic behavior for $N \rightarrow \infty$ of the average effective resistance of graphs sampled from a piecewise Lipschitz graphon bounded away from zero, we can see that:

$$
N R_{N}^{\text {ave }}=\sum_{i=2}^{N} \frac{1}{\lambda_{i}} \leq(N-1) \frac{1}{\lambda_{2}} \leq \frac{1}{\mu_{2}} .
$$

As noticed in Remark 2. Lemma 3 implies that $\left|\mu_{2}-\bar{\mu}_{2}\right|$ goes to zero a.s. Moreover, by Lemma $5 \bar{\mu}_{2} \geq \eta_{W}>0$. From this, we can conclude that almost surely also $\mu_{2}$ remains bounded away from zero, and hence $R_{N}^{\text {ave }}=O(1 / N)$ a.s. Also, since $\lambda_{N} \leq N$ we get:

$$
N R_{N}^{\text {ave }} \geq(N-1) \frac{1}{N} .
$$

Therefore $R_{N}^{\text {ave }}=\Theta(1 / N)$ a.s.

Considering that the distribution of the eigenvalues of the Laplacian matrix is similar to the distribution of the degrees, we can estimate the average effective resistance $R_{N}$ of a simple graph $G_{N}$ through the degree function of the graphon $W$, by defining an analogous quantity as:

$$
R_{W, N}^{\text {ave }}:=\frac{1}{N} \int_{0}^{1} \frac{1}{d(x)} \mathrm{d} x .
$$

Theorem 2. For a piecewise Lipschitz graphon $W$ with minimum $\eta_{W}>0$ and for $N$ satisfying conditions (4a), (4b), (4c) and condition:

$$
\frac{\log (2 N / \nu)}{N}<\frac{\eta_{W}^{2}}{1+2 \eta_{W}},
$$

let $R_{N}^{\text {ave }}$ be the average effective resistance of a graph $G_{N}$ sampled from $W$. Then, with probability at least $1-3 \nu$ :

$$
\begin{aligned}
& \left|R_{N}^{\text {ave }}-R_{W, N}^{\text {ave }}\right| \leq \\
& \frac{1}{N\left(\eta_{W}-\gamma(N)\right)}\left(\frac{1}{N}+\frac{\phi(N)}{\delta_{W}}+\frac{\sqrt[4]{2} \sqrt{\left\|\mid T_{W}\right\|+\phi(N)}}{N^{1 / 4}\left(\eta_{W}-\varphi(N)\right)}\right),
\end{aligned}
$$

with $\phi(N)$ as in Lemma 2 and $\varphi(N)$ and $\gamma(N)$ as in Lemma 3

Proof. In this proof, we will make use of the bounds (7), 8), (13) and (14) from Lemmas 2 and 3 by Lemma 4 the event that all four bounds hold true has probability at least $1-3 \nu$.

If we define the step function

$$
r_{N}(x)=\sum_{i=2}^{N} \frac{1}{\lambda_{i}} \mathbb{1}_{B_{i}^{N}}(x),
$$


it is easy to see that $R_{N}^{\text {ave }}=\left\|r_{N}(x)\right\|_{1}$ and $R_{W, N}^{\text {ave }}=$ $\left\|\frac{1}{N d(x)}\right\|_{1}$, so that we have:

$$
\begin{aligned}
& \left|R_{N}^{\text {ave }}-R_{W, N}^{\text {ave }}\right|=\left|\left\|r_{N}(x)\right\|_{1}-\left\|\frac{1}{N d(x)}\right\|_{1}\right| \\
\leq & \left|\left\|r_{N}(x)\right\|_{1}-\left\|\frac{1}{N d_{N}(x)}\right\|_{1}\right|+\left|\left\|\frac{1}{N d_{N}(x)}\right\|_{1}-\left\|\frac{1}{N d(x)}\right\|_{1}\right| .
\end{aligned}
$$

We start by studying the first term in (23). We notice that

$$
\left\|\frac{1}{N d_{N}(x)}\right\|_{1}=\frac{1}{N} \sum_{i=1}^{N} \frac{1}{d_{i}}=\frac{1}{N} \sum_{i=1}^{N} \frac{1}{d_{(i)}}=\left\|\frac{1}{N \tilde{d}_{N}(x)}\right\|_{1} .
$$

We use this remark and the reverse triangle inequality (i.e., $|\|x\|-\|y\|| \leq\|x-y\|)$, to obtain

$$
\|\| r_{N}(x)\left\|_{1}-\right\| \frac{1}{N d_{N}(x)}\left\|_{1} \mid \leq\right\| r_{N}(x)-\frac{1}{N \tilde{d}_{N}(x)} \|_{1} .
$$

Then, we have:

$$
\begin{aligned}
\left\|r_{N}(x)-\frac{1}{N \tilde{d}_{N}(x)}\right\|_{1} & =\frac{1}{N^{2} \delta_{(1)}}+\frac{1}{N} \sum_{i=2}^{N}\left|\frac{d_{(i)}-\lambda_{i}}{d_{(i)} \lambda_{i}}\right| \\
& \leq \frac{1}{N^{2} \delta_{(1)}}+\frac{\sum_{i=2}^{N}\left|d_{(i)}-\lambda_{i}\right|}{N^{3} \mu_{2} \delta_{(1)}} .
\end{aligned}
$$

By using the norm inequality $\|\cdot\|_{1} \leq \sqrt{N}\|\cdot\|_{2}$ and applying Wielandt-Hoffman Theorem to $A_{N}=D_{N}-L_{N}$, we obtain:

$$
\sum_{i=2}^{N}\left|d_{(i)}-\lambda_{i}\right| \leq \sqrt{N}\left(\sum_{i=2}^{N}\left|d_{(i)}-\lambda_{i}\right|^{2}\right)^{1 / 2} \leq \sqrt{N}\left\|A_{N}\right\|_{F} .
$$

Finally, we can use Lemma 1 and (7) to obtain:

$$
\left\|r_{N}(x)-\frac{1}{N \tilde{d}_{N}(x)}\right\|_{1} \leq \frac{1}{N^{2} \delta_{(1)}}+\frac{\sqrt[4]{2} \sqrt{\left\|T_{W}\right\|+\phi(N)}}{N^{5 / 4} \mu_{2} \delta_{(1)}} .
$$

Now we study the second term in 23. We use again the reverse triangle inequality and Hölder's inequality so that we have:

$$
\left\|\frac{1}{N d_{N}(x)}\right\|_{1}-\left\|\frac{1}{N d(x)}\right\|_{1} \mid \leq\left\|\frac{1}{N d_{N}(x)}-\frac{1}{N d(x)}\right\|_{2} .
$$

Then we obtain:

$$
\begin{gathered}
\left\|\frac{1}{N d_{N}(x)}-\frac{1}{N d(x)}\right\|_{2}=\left(\int_{0}^{1}\left|\frac{d(x)-d_{N}(x)}{N d(x) d_{N}(x)}\right|^{2} \mathrm{~d} x\right)^{1 / 2} \\
\leq \frac{1}{N \delta_{(1)} \delta_{W}}\left(\int_{0}^{1}\left|d(x)-d_{N}(x)\right|^{2} \mathrm{~d} x\right)^{1 / 2} .
\end{gathered}
$$

Since $\left(\int_{0}^{1}\left|d(x)-d_{N}(x)\right|^{2} \mathrm{~d} x\right)^{1 / 2}=\left\|d_{N}(x)-d(x)\right\|_{2}$, we can apply 13 and get:

$$
\left\|\frac{1}{N d_{N}(x)}\right\|_{1}-\left\|\frac{1}{N d(x)}\right\|_{1} \mid \leq \frac{1}{N \delta_{(1)} \delta_{W}} \phi(N) .
$$

Using the bounds obtained for the two terms in 23, we get:

$$
\left|R_{N}^{\mathrm{ave}}-R_{W, N}^{\mathrm{ave}}\right| \leq \frac{1}{N^{2} \delta_{(1)}}+\frac{\sqrt[4]{2} \sqrt{\left\|T_{W}\right\| \mid+\phi(N)}}{N^{5 / 4} \mu_{2} \delta_{(1)}}+\frac{\phi(N)}{N \delta_{(1)} \delta_{W}} .
$$

By using (13) and (14) we obtain:

$$
\begin{aligned}
\left|R_{N}^{\text {ave }}-R_{W, N}^{\text {ave }}\right| & \leq \frac{1}{N^{2}\left(\bar{\delta}_{(1)}-\gamma(N)\right)}+\frac{\phi(N)}{N \delta_{W}\left(\bar{\delta}_{(1)}-\gamma(N)\right)} \\
& +\frac{\sqrt[4]{2} \sqrt{\left\|T_{W}\right\|+\phi(N)}}{N^{5 / 4}\left(\bar{\delta}_{(1)}-\gamma(N)\right)\left(\bar{\mu}_{2}-\varphi(N)\right)}
\end{aligned}
$$

Finally we get the desired result by using $\delta_{(1)} \geq \eta_{W}$ and Lemma 5. Notice that assumption $\eta_{W}>0$ and condition (22) ensure that the denominators appearing in the upper bound are positive.

Remark 4. Theorem 2 gives an upper bound on the absolute error $\left|R_{N}^{\text {ave }}-R_{W, N}^{\text {ave }}\right|$. This bound holds true with probability at least $1-3 \nu$, and has an expression which depends both on $\nu$ and on $N$. We are interested in its asymptotic behaviour for $N \rightarrow \infty$. When we consider a constant $\nu$ or $\nu=1 / N^{\alpha}$, this upper bound goes to zero as $O\left(\left(\log (N) / N^{5}\right)^{1 / 4}\right)$, and with the further assumption that the graphon is Lipschitz $(K=0)$, it decays as $O\left((1 / N)^{5 / 4}\right)$. By choosing $\alpha>1$, we can then apply Borel-Cantelli Lemma, and obtain that almost surely $\left|R_{N}^{\text {ave }}-R_{W, N}^{\text {ave }}\right|$ decays to zero as $O\left(\left(\log (N) / N^{5}\right)^{1 / 4}\right)$, and moreover as $O\left((1 / N)^{5 / 4}\right)$ in case the graphon is Lipschitz. It is also interesting to study the relative error $\left|R_{N}^{\text {ave }}-R_{W, N}^{\text {ave }}\right| / R_{N}^{\text {ave }}$. Recalling Remark 3 about the asymptotic behaviour of the denominator, we obtain that the relative error almost surely decays to zero as $O\left((\log (N) / N)^{1 / 4}\right)$, and as $O\left((1 / N)^{1 / 4}\right)$ if the graphon is Lipschitz.

\subsection{Deterministic Sampling}

An alternative procedure for the generation of complete weighted graphs from graphons is the use of deterministic latent variables proposed in [32], such that the adjacency matrix of $\bar{G}_{N}$ is generated as:

$$
\bar{A}_{N}(i, j)=W(i / N, j / N) \quad \text { for all } i, j \in\{1, \ldots, N\} .
$$

All the results of Section 3 (with exception of Proposition 4 easily extend to deterministic sampling with minor adjustments, which we detail here. Lemma 3 and Lemma 5 do not depend on the sampling method and remain the same. As indicated in [32], the factor $b_{N}$ of $\vartheta(N)$ and $\phi(N)$ in Lemma 2 is redefined as $b_{N}:=1 / N$ and (5) and (6) hold with probability 1 while (7) and (8) hold with probability at least $1-\nu$. With the new definition of $b_{N}$, Propositions 1 and 2 hold with probability at least $1-\nu$ and Lemma 4 and Theorems 1 and 2 hold with probability at least $1-2 \nu$. The rates of convergence in Remarks 2 and 3 do not change while for Remarks 1 and 4 the rate of convergence for piecewise Lipschitz and Lipschitz graphons is $O\left(1 / N^{1 / 4}\right)$.

Proposition 4 instead cannot be easily extended to the deterministic case because in [14] the compact convergence of the operators is proved by using Glivenko-Cantelli Theorem in one of the steps, which is formulated for random variables.

\section{NUMERICAL EXAMPLE}

We consider the graphon $W(x, y)=1-0.8 x y$, which is Lipschitz and bounded away from zero (its minimum is $\eta_{W}=0.2$ ). Its degree function is $d(x)=1-0.4 x$, whose minimum is $\delta_{W}=0.6$. To validate the results, we consider a 


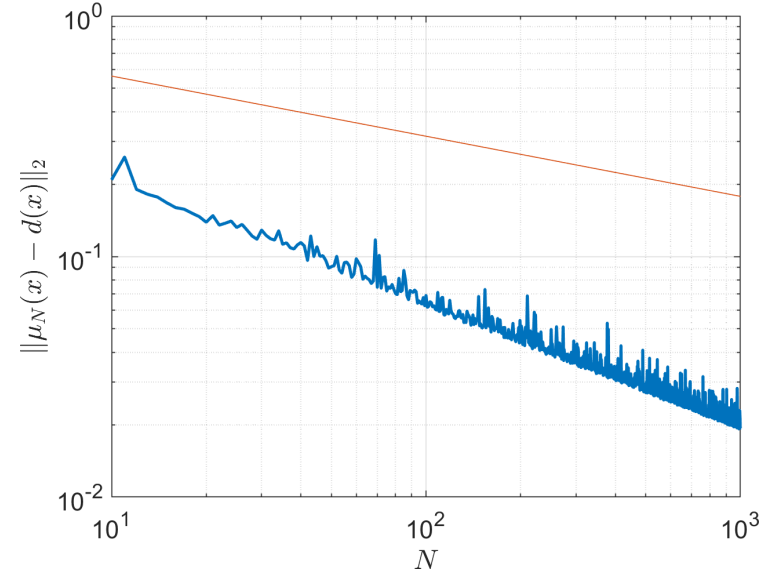

Fig. 1. $\left\|\mu_{N}(x)-d(x)\right\|_{2}$ for growing $N$. Its decay is consistent with the upper bound $O\left(N^{-1 / 4}\right)$, depicted in red.

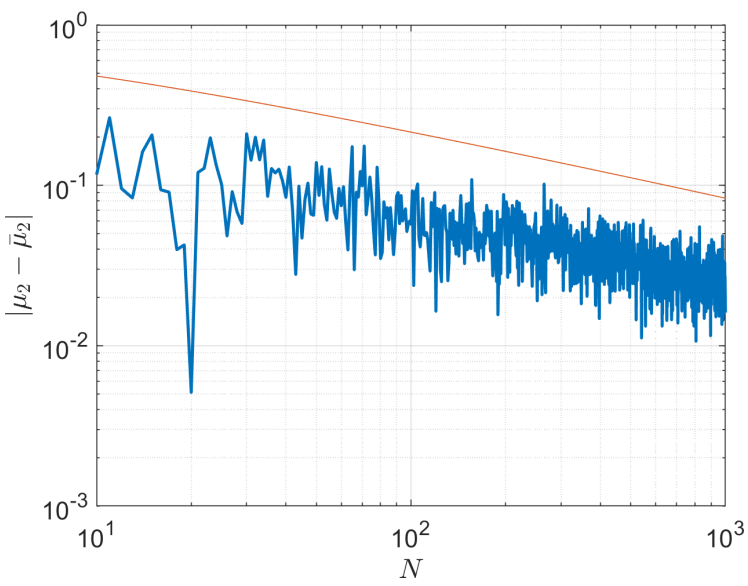

Fig. 2. $\left|\mu_{2}-\bar{\mu}_{2}\right|$ for growing $N$. Its decay is comparable with the upper bound $(\log (N) / N)^{1 / 2}$, depicted in red.

sequence of simple graphs that are randomly sampled from $W$ for $10 \leq N \leq 1000$. Fig. 1 presents the approximation of the distribution of the normalized eigenvalues by using the degree function of the graphon.

By solving the eigenfunction equation (i.e., $\left(\mathcal{L}_{W} \psi\right)(x)=$ $\kappa \psi(x))$ we find that the operator only has the trivial eigenvalue $\kappa_{1}=0$, so that, by Proposition 4 and Remark 2 a.s. $\lim \bar{\mu}_{2}=\lim \mu_{2}=\delta_{W}$. Fig. 2 shows the difference between the second normalized eigenvalues of $G_{N}$ and $\bar{G}_{N}$ while Fig. 3 illustrates their convergence towards $\delta_{W}$ as per Proposition 4 . We can observe that the convergence of $\mu_{2}$ is slower than the convergence of $\bar{\mu}_{2}$.

The approximation of the average effective resistance is performed through:

$$
R_{W, N}^{\mathrm{ave}}=\frac{1}{N} \int_{0}^{1} \frac{\mathrm{d} x}{1-0.4 x}=-\frac{5}{2 N} \log (0.6) .
$$

Fig. 4 shows the approximation of the average effective resistance: the relative error plot suggests our bound on the convergence rate to be tight in its dependence on $N$.

\section{CONCLUSION AND FUTURE WORK}

In this paper, the spectrum of the Laplacian matrix of a network sampled from a graphon was analyzed using the

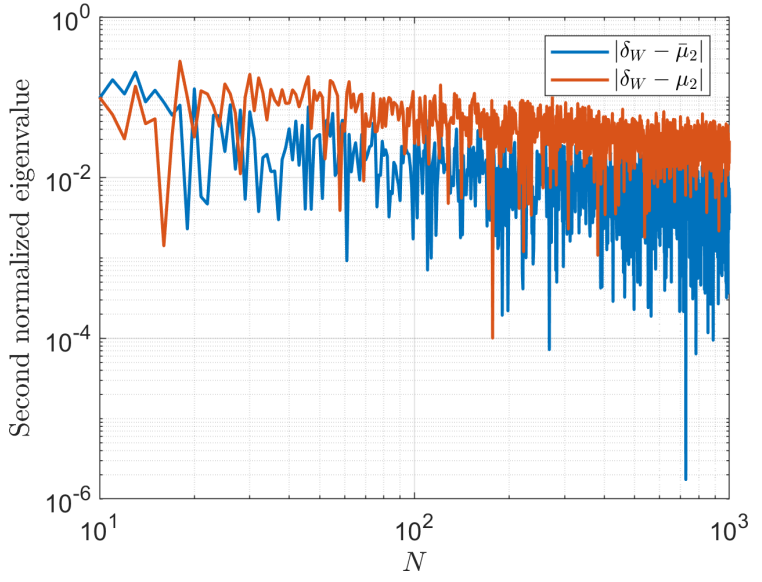

Fig. 3. Convergence of second normalized eigenvalues for growing $N$.

degree function of the graphon. First, we showed that for networks derived from a graphon, the distribution of the eigenvalues of the Laplacian matrix is determined mainly by the degrees of the network. Then, we showed that the average effective resistance of a graph sampled from a graphon can be estimated by using the degree function of the graphon. For both problems, we have derived explicit bounds on the approximation error.

Even if this paper has shown some initial applications of the graphon Laplacian operator, numerous related questions remain open. Indeed, our methods can be applied to estimate other functions of the Laplacian spectrum, such as the spectral zeta function [38] and several performance metrics in estimation and control problems over networks [37], [39]. Furthermore, the graphon Laplacian operator could be used to define suitable infinite-dimensional dynamical systems that approximate dynamical systems on finite-dimensional graphs, as done in [10] with the normalized Laplacian and in [9], [40] with the adjacency matrix. This line of work entails some technical difficulties, such as the lack of compactness of the graphon Laplacian operators.

Finally, we ought to recall that graphons are limited to approximate dense graphs, whereas many relevant networks are sparse. It is therefore an open question to develop the suitable tools to address these cases.

\section{References}

[1] L. Lovász and B. Szegedy, "Limits of dense graph sequences," Journal of Combinatorial Theory, Series B, vol. 96, no. 6, pp. 933-957, 2006.

[2] L. Lovász, Large networks and graph limits. American Mathematical Soc., 2012, vol. 60 .

[3] S. Janson, "Graphons, cut norm and distance, couplings and rearrangements," New York J. Math. Monographs, vol. 4, pp. 1-76, 2013.

[4] F. Parise and A. Ozdaglar, "Graphon games," in Proceedings of the 2019 ACM Conference on Economics and Computation. ACM, 2019, pp. 457-458.

[5] S. Gao, P. E. Caines, and M. Huang, "LQG graphon mean field games," arXiv preprint arXiv:2004.00679, 2020.

[6] L. Ruiz, L. F. Chamon, and A. Ribeiro, "The graphon Fourier transform," arXiv preprint arXiv:1910.10195, 2019.

[7] — "Graphon signal processing," arXiv preprint arXiv:2003.05030, 2020. 

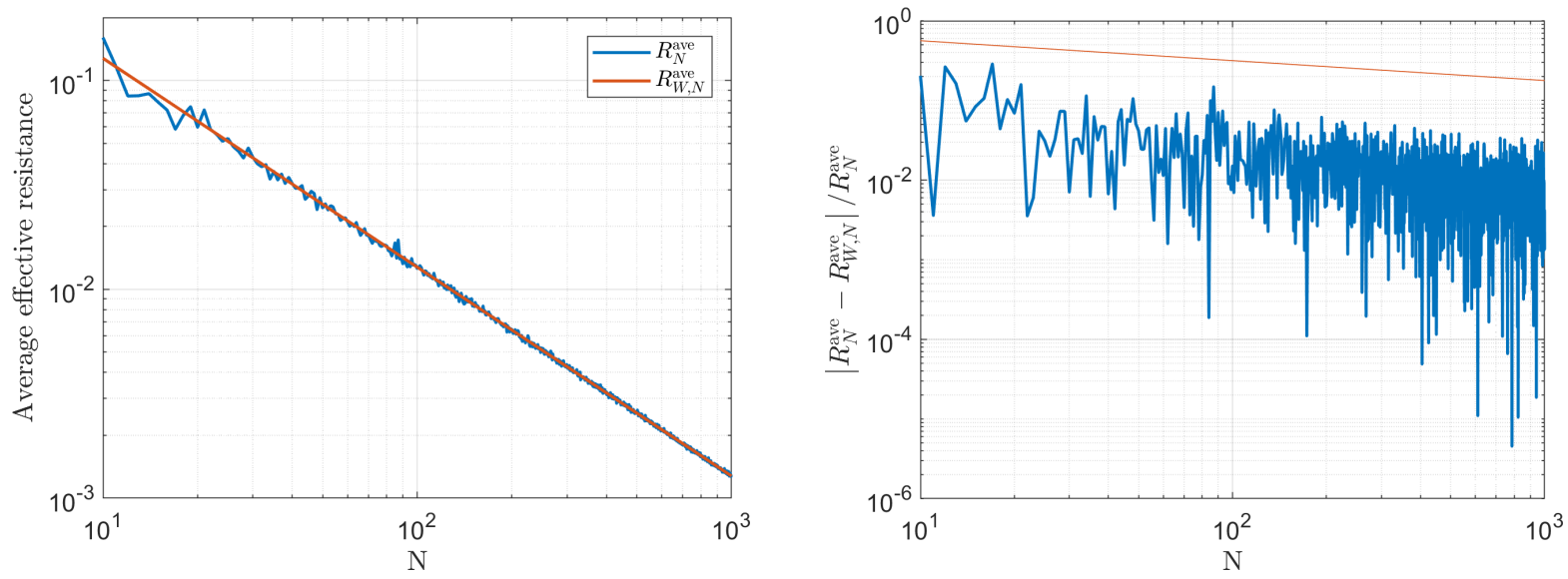

Fig. 4. Left: Average effective resistance for growing $N$. Right: relative error $\left|R_{N}^{\text {ave }}-R_{W, N}^{\text {ave }}\right| / R_{N}^{\text {ave }}$ for growing $N$. Its decay is comparable with $N^{-1 / 4}$, depicted in red.

[8] S. Gao and P. E. Caines, "Optimal and approximate solutions to linear quadratic regulation of a class of graphon dynamical systems," in IEEE Conference on Decision and Control, 2019, pp. 8359-8365.

[9] - "Graphon control of large-scale networks of linear systems," IEEE Transactions on Automatic Control, vol. 65, no. 10, pp. 40904105, 2020.

[10] J. Petit, R. Lambiotte, and T. Carletti, "Random walks on dense graphs and graphons," arXiv preprint arXiv:1909.11776, 2019.

[11] S. Gao and P. E. Caines, "Spectral representations of graphons in very large network systems control," in IEEE Conference on Decision and Control, 2019, pp. 5068-5075.

[12] R. Vizuete, P. Frasca, and F. Garin, "Graphon-based sensitivity analysis of SIS epidemics," IEEE Control Systems Letters, vol. 4, no. 3, pp. 542-547, 2020.

[13] F. Bullo, Lectures on Network Systems. Kindle Direct Publishing, 2019, with contributions by J. Cortes, F. Dorfler, and S. Martinez. [Online]. Available: http://motion.me.ucsb.edu/book-lns

[14] U. von Luxburg, M. Belkin, and O. Bousquet, "Consistency of spectral clustering," The Annals of Statistics, pp. 555-586, 2008.

[15] B. Mohar and S. Poljak, "Eigenvalues in combinatorial optimization," in Combinatorial and graph-theoretical problems in linear algebra. Springer, 1993, pp. 107-151.

[16] D. I. Shuman, S. K. Narang, P. Frossard, A. Ortega, and P. Vandergheynst, "The emerging field of signal processing on graphs: Extending high-dimensional data analysis to networks and other irregular domains," IEEE Signal Processing Magazine, vol. 30, no. 3, pp. 83-98, 2013.

[17] F. Dörfler, J. W. Simpson-Porco, and F. Bullo, "Electrical networks and algebraic graph theory: Models, properties, and applications," Proceedings of the IEEE, vol. 106, no. 5, pp. 977-1005, 2018.

[18] P. G. Doyle and J. L. Snell, Random Walks and Electric Networks, ser. Carus Monographs. Mathematical Association of America, 1984.

[19] L. Lovász, "Random walks on graphs: A survey," Combinatorics, Paul Erdos is Eighty, vol. 2, no. 1, pp. 1-46, 1993.

[20] E. Lovisari, F. Garin, and S. Zampieri, "Resistance-based performance analysis of the consensus algorithm over geometric graphs," SIAM Journal on Control and Optimization, vol. 51, no. 5, pp. 3918-3945, 2013.

[21] R. Vizuete, P. Frasca, and E. Panteley, "On the influence of noise in randomized consensus algorithms," IEEE Control Systems Letters, vol. 5, no. 3, pp. 1025-1030, 2021.

[22] P. Barooah and J. P. Hespanha, "Estimation on graphs from relative measurements," IEEE Control Systems Magazine, vol. 27, no. 4, pp. $57-74,2007$.

[23] W. S. Rossi, P. Frasca, and F. Fagnani, "Average resistance of toroidal graphs," SIAM Journal on Control and Optimization, vol. 53, no. 4, pp. 2541-2557, 2015.

[24] A. K. Chandra, P. Raghavan, W. L. Ruzzo, R. Smolensky, and P. Tiwari, "The electrical resistance of a graph captures its commute and cover times," Computational Complexity, vol. 6, no. 4, pp. 312340, 1996.
[25] Y. Saad, Numerical Methods for Large Eigenvalue Problems. Society for Industrial and Applied Mathematics, 2011.

[26] N. K. Vishnoi, "Laplacian solvers and their algorithmic applications," Theoretical Computer Science, vol. 8, no. 1-2, pp. 1-141, 2012.

[27] D. A. Spielman and S.-H. Teng, "Nearly linear time algorithms for preconditioning and solving symmetric, diagonally dominant linear systems," SIAM Journal on Matrix Analysis and Applications, vol. 35, no. 3, pp. 835-885, 2014.

[28] R. Kyng and S. Sachdeva, "Approximate Gaussian elimination for Laplacians-fast, sparse, and simple," in 2016 IEEE 57th Annual Symposium on Foundations of Computer Science (FOCS). IEEE, 2016, pp. 573-582.

[29] U. von Luxburg, O. Bousquet, and M. Belkin, "Limits of spectral clustering," in Advances in neural information processing systems, 2005, pp. 857-864.

[30] C. Zhan, G. Chen, and L. F. Yeung, "On the distributions of Laplacian eigenvalues versus node degrees in complex networks," Physica A: Statistical Mechanics and its Applications, vol. 389, no. 8, pp. 1779-1788, 2010.

[31] S. Hata and H. Nakao, "Localization of Laplacian eigenvectors on random networks," Scientific reports, vol. 7, no. 1, p. 1121, 2017.

[32] M. Avella-Medina, F. Parise, M. T. Schaub, and S. Segarra, "Centrality measures for graphons: Accounting for uncertainty in networks," IEEE Transactions on Network Science and Engineering, vol. 7, no. 1, pp. 520-537, 2020.

[33] R. A. Horn and C. R. Johnson, Matrix analysis. Cambridge university press, 2012.

[34] F. Chung and M. Radcliffe, "On the spectra of general random graphs," Electronic Journal of Combinatorics, vol. 18, no. 1, p. 215 , 2011.

[35] P. Diaconis and D. Stroock, "Geometric bounds for eigenvalues of Markov chains," Annals of Applied Probability, vol. 1, no. 1, pp. 36-61, 1991.

[36] F. Chung, "The diameter and Laplacian eigenvalues of directed graphs," Electronic Journal of Combinatorics, vol. 13, no. 1, 2006.

[37] F. Fagnani and P. Frasca, "Averaging with exogenous inputs and electrical networks," in Introduction to Averaging Dynamics over Networks. Springer, 2018, pp. 109-131.

[38] A. Karlsson, "Spectral zeta functions," in Discrete and Continuous Models in the Theory of Networks. Cham: Springer International Publishing, 2020, pp. 199-211.

[39] F. Garin and L. Schenato, "A survey on distributed estimation and control applications using linear consensus algorithms," in Networked control systems. Springer, 2010, pp. 75-107.

[40] J.-F. Delmas, D. Dronnier, and P.-A. Zitt, “An infinite-dimensional SIS model," arXiv preprint arXiv:2006.08241, 2020. 


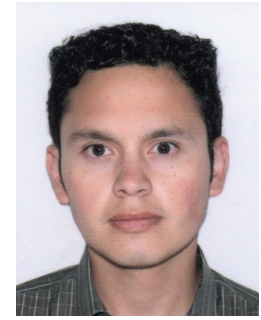

Renato Vizuete received the B.S. degree (summa cum laude) in Electronics and Control Engineering from Escuela Politécnica Nacional, Ecuador and the M.S. degree (très bien) in Systems, Control and Information Technologies from Université Grenoble Alpes, France. He was the recipient of the Persyval-Lab Excellence Master Scholarship from Université Grenoble Alpes in 2018 . He is currently a PhD student at L2S CentraleSupélec and GIPSA-lab, France. His research interests include: control theory, multiagent systems, hybrid systems and networked control systems.

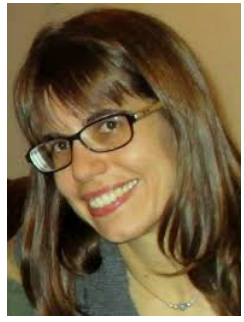

Federica Garin (M'16) is a researcher with the NeCS team at INRIA and GIPSA-lab, Grenoble (France). She received her B.S., M.S., and Ph.D. degrees in Applied Mathematics from Politecnico di Torino (Italy) in 2002, 2004, and 2008, respectively. She was a post-doctoral researcher at Università di Padova (Italy) in 2008 and 2009, and at INRIA Grenoble (France) in 2010. She is an Associate Editor in the IEEE-CSS Conference Editorial Board and in the European Control Association (EUCA) Conference Editorial Board. Her current research interests are in distributed algorithms and network control systems.

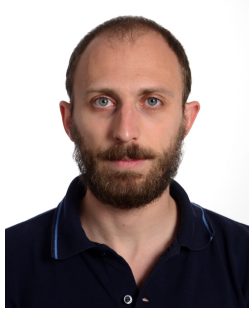

Paolo Frasca (M'13, SM'18) received the Ph.D. degree in Mathematics for Engineering Sciences from Politecnico di Torino, Torino, Italy, in 2009. From 2013 to 2016, he was an Assistant Professor at the University of Twente in Enschede, the Netherlands. Since October 2016 he is a CNRS Researcher affiliated with GIPSA-lab, Grenoble, France. His research interests are in the theory of networks and control systems, with main applications to transportation and social networks. 